\title{
Bisphenol A reactions with hydroxyl radicals: diverse pathways determined between deionized water and tertiary treated wastewater solutions
}

\author{
Julie R. Peller $\cdot$ Stephen P. Mezyk $\cdot$ William J. Cooper
}

Received: 26 July 2007 / Accepted: 2 October 2007 / Published online: 15 January 2009

(C) The Author(s) 2009. This article is published with open access at Springerlink.com

\begin{abstract}
The oxidative reactions of bisphenol A (BPA) with radiolytically generated hydroxyl radicals were studied in both deionized water and tertiary treated wastewaters. In deionized water, bisphenol A reacts with the hydroxyl radical by addition to the aromatic ring, $\mathrm{k}=6.9 \times 10^{9}( \pm 0.2) \mathrm{M}^{-1} \mathrm{~s}^{-1}$, to eventually form the prominent, long-lived, hydroxylated intermediate product. In contrast, in tertiary treated water solutions, although the initial hydroxyl radical addition reaction occurs, the hydroxylation is averted, and a different mechanistic pathway ensues. The removal constant for the hydroxyl radical reaction with BPA is $0.45 \pm 0.04 \mu \mathrm{mol} / \mathrm{kGy}$, corresponding to an overall degradation efficiency of $76 \%$.
\end{abstract}

Keywords Bisphenol A - Deionized water - Hydroxyl radical reaction .

Tertiary treated wastewaters

\section{Introduction}

Bisphenol A (2,2-bis-(4-hydroxyphenyl)propane, BPA) is an industrial chemical that is currently receiving a great deal of attention as an environmental contaminant. BPA is the key chemical used in the manufacture of polycarbonates and epoxy

J. R. Peller $(\bowtie)$

Department of Chemistry, Indiana University Northwest, 3400 Broadway, Gary, IN 46408, USA

e-mail: jpeller@iun.edu

S. P. Mezyk

Department of Chemistry and Biochemistry, California State University at Long Beach, 1250 Bellflower Blvd, Long Beach, CA 90840, USA

W. J. Cooper

Urban Water Research Center, Department of Civil and Environmental Engineering,

University of California, Irvine, Irvine, CA 92697-2175, USA 
resins, materials that are utilized in a number of applications such as optical recording media, sports equipment, medical equipment, and beverage containers. Due to the massive quantities of BPA utilized in industry, measurable amounts of the compound have been reported in various water supplies and wastewaters [1-5]. The mixing of industrial wastewaters with sewage effluent and other bodies of water is the most likely means for BPA contamination of natural water systems.

Bisphenol $\mathrm{A}$ is classified as an endocrine disruptor, a compound which mimics natural hormones, leading to disruptive development and reproduction. The presence of endocrine disrupting compounds in natural waters is believed to cause sexual disruption in aquatic organisms [6,7]. Human exposure is most likely the result of the consumption of tainted water or from the leaching of plastic consumer products. Even very low level exposure of bisphenol A has been suspected in abnormal development in many different organisms [8-10].

Investigations into the removal of bisphenol A from aqueous environments have studied the use of carbon adsorption [11], electrochemical treatments [12], oxidative coupling mediated by horseradish peroxidase [13], as well as photocatalytic oxidation [14-16], sonochemical oxidation [17], ozone oxidation [18], and enzymatic oxidation [19]. While each process offers some degree of effectiveness in the breakdown or removal of BPA in aqueous solutions, advanced oxidation processes (AOPs) such as radiolysis, photocatalysis, sonolysis, and UV-peroxide are often considered the best choice for the removal of recalcitrant compounds. These systems rely on the in-situ production of hydroxyl radicals ( $\mathrm{OH})$, which destructively react with many chemical moieties [20].

Phenolic compounds, such as bisphenol A, are readily oxidized by the hydroxyl radical [21-24]. The typical reaction between an aromatic compound and the hydroxyl radical consists of $\mathrm{OH}$ addition to the electron rich ring. The hydroxylated intermediates often react more rapidly in subsequent reactions with the hydroxyl radical, but can also be more resistant to further oxidation. Therefore, to ensure quantitative removal of contaminants by this methodology, it is necessary to understand all of the chemical pathways involved in these hydroxyl radical reactions.

Typical laboratory investigations into the chemical details of AOP remediation techniques employ deionized water as the reaction solvent. While this allows for unambiguous measurements for the compound of interest with the reactive oxidant, real water conditions can be significantly different. In this study, the chemistry involved in the oxidative degradation of BPA, in both deionized water and in tertiary treated wastewater matrices, was investigated using radiolysis techniques. Secondary or tertiary treatment of wastewater is often the final treatment step before disinfection (chlorination, UV, or ozonation). Tertiary treatment may involve sand filtration, activated carbon, and/or other processes, but most tertiary treatment processes are not capable of removing all of the dissolved organics, which may include pharmaceuticals, pesticides, and other household and industrial chemicals. Many wastewater treatment plants incorporate post-tertiary treatments such as membrane filtration and/or reverse osmosis to eliminate these dissolved compounds. Six different treated wastewater samples from two different water treatment plants were used in this study (Tables 1 and 2). 
Table 1 Selected water quality values for the three different water samples from Scottsdale, Arizona, Wastewater Treatment Plant used in the experiments

\begin{tabular}{llll}
\hline Parameters & Before membrane filtration & Membrane effluent & RO effluent \\
\hline TOC $(\mathrm{mg} / \mathrm{L})$ & 7.8 & 6.8 & $<0.2$ \\
Total alkalinity $(\mathrm{mg} / \mathrm{L})$ & 158 & 160 & $<10$ \\
Bicarbonate & 158 & 160 & $<10$ \\
Chloride (mg/L) & 340 & 340 & 4.7 \\
pH & 7.5 & 7.3 & 5.6 \\
Total hardness & 364 & 361 & 2.1 \\
Sulfates & 270 & 270 & 1.2 \\
Nitrates & 4.9 & 4.8 & 0.16 \\
\hline
\end{tabular}

Table 2 Selected water quality values for the three different water samples from the Orange County, CA, Wastewater Treatment Pilot Plant used in the experiments

\begin{tabular}{lllll}
\hline Parameters & Q1 & ROA & ROB & Deionized \\
\hline TOC $(\mathrm{mg} / \mathrm{L})$ & 15 & 11.9 & 0.3 & $<0.2$ \\
Total alkalinity $(\mathrm{mg} / \mathrm{L})$ & 287 & - & 12.3 & 0 \\
Bicarbonate & 158 & - & 15 & 0 \\
Chloride (mg/L) & 223 & 4.5 & 4.4 & 0 \\
$\mathrm{pH}$ & 7.8 & 6.9 & 6.9 & 7.0 \\
Organic N (mg/L) & 2.9 & - & 0.5 & 0 \\
Sulfates (mg/L) & 279 & 130 & 3.2 & 0 \\
\hline
\end{tabular}

By exposing bisphenol A solutions to ionizing radiation, we have determined the kinetics of its hydroxyl radical degradation and identified different reaction pathways for deionized and treated wastewaters solvents.

\section{Experimental}

The chemicals used in this study were obtained from the Aldrich Chemical Company and of the highest purity available, and used as received. Solutions were made using water filtered by a Millipore Milli-Q system or in treated wastewater. All solutions were completely saturated with high purity $\mathrm{N}_{2} \mathrm{O}$, for hydroxyl radical experiments, to remove dissolved oxygen, ensuring only oxidizing conditions.

Treated wastewater samples used in this study were provided by Orange County, CA, Sanitation District, Interim Water Factory 21 and by the City of Scottsdale, Arizona, Water Treatment Plant. Selected water quality parameters are shown in Tables 1 and 2 .

Electron pulse radiolysis

The linear accelerator (LINAC) electron pulse radiolysis system at the Radiation Laboratory, University of Notre Dame, was used for the reaction rate constant and 
transient spectra determinations. This system has previously been described in detail [25].

During rate constant measurements, the solution vessels were continuously sparged with nitrous oxide gas. Solution flow rates in these experiments were adjusted so that each irradiation was performed on a fresh sample. Dosimetry [26] was performed using $\mathrm{N}_{2} \mathrm{O}$-saturated, $1.00 \times 10^{-2} \mathrm{M}$ KSCN solutions at $\lambda=475 \mathrm{~nm},\left(\mathrm{G}_{\varepsilon}=5.2 \times 10^{-4} \mathrm{~m}^{2} \mathrm{~J}^{-1}\right)$ with average doses of 3-5 Gy per 2-3 ns pulse. Throughout this article, $\mathrm{G}$ is defined in $\mu \mathrm{mol} \mathrm{J}^{-1}$, and $\varepsilon$ is in units of $\mathrm{M}^{-1} \mathrm{~cm}^{-1}$. All kinetics and transient experiments were performed at ambient temperature $\left(21 \pm 1^{\circ} \mathrm{C}\right)$ and in natural, unadjusted $\mathrm{pH}$ solution.

Steady state experiments

The ${ }^{60} \mathrm{Co} \gamma$ irradiators used were also at the Radiation Laboratory, and consisted of: a Shepherd 109, with a dose rate of $0.889 \mathrm{kGy} \mathrm{min}^{-1}$; a Shepherd 109 with a dose rate of $0.153 \mathrm{kGy} \mathrm{min}^{-1}$; and a Gammacell 220, with a dose rate of $0.0501 \mathrm{kGy} \mathrm{min}^{-1}$. For purely oxidative conditions, aqueous solutions were again pre-saturated with $\mathrm{N}_{2} \mathrm{O}(\mathrm{g})$ before irradiation.

The loss of the bisphenol A and the formation of intermediates were followed using a Waters HPLC system (Millennium 2010, Waters 717 plus Autosampler, Waters 600 Controller Solvent Pump) equipped with a Supelco Discovery ${ }^{\circledR}$ C18 column, $5 \mu \mathrm{m}(250 \times 4.6 \mathrm{~mm})$. A gradient solvent flow utilized water and methanol and acetic acid solution (1\%), where the initial solvent mixture was $70 \%$ methanol and $30 \%$ water. At the 13-min mark, the solvent flow changed to $30 \%$ methanol, $68 \%$ water, and $2 \%$ acetic acid solution. The solvent switched back to the original flow by $17 \mathrm{~min}$. A photodiode array detector monitored the $200-400 \mathrm{~nm}$ range.

Identification of intermediates

Intermediates were identified by GC/MS (UCI) and LC/MS analyses.

\section{Results and discussion}

For both the electron pulse and $\gamma$-radiolysis experiments of this study, the irradiation of water solutions forms free radicals according to the reaction [20]:

$$
\mathrm{H}_{2} \mathrm{O}--\mathrm{WW} \rightarrow[0.28]^{\circ} \mathrm{OH}+[0.06]^{\circ} \mathrm{H}+[0.27] \mathrm{e}_{\mathrm{aq}}^{-}+[0.05] \mathrm{H}_{2}+[0.07] \mathrm{H}_{2} \mathrm{O}_{2}+[0.27] \mathrm{H}^{+}
$$

The numbers in brackets are the G-values (yields) in $\mu \mathrm{mol} / \mathrm{Gy}$ of energy.

To establish oxidizing conditions involving only the hydroxyl radical, the water solutions were saturated with $\mathrm{N}_{2} \mathrm{O}(\mathrm{g})$, which quantitatively converts the hydrated electrons, $\mathrm{e}_{\mathrm{aq}}^{-}$, and hydrogen atoms, ${ }^{\bullet} \mathrm{H}$, to hydroxyl radicals [20]

$$
\begin{gathered}
\mathrm{e}_{\mathrm{aq}}^{-}+\mathrm{N}_{2} \mathrm{O}+\mathrm{H}_{2} \mathrm{O} \rightarrow \mathrm{N}_{2}+\mathrm{OH}^{-}+\bullet \mathrm{OH} \quad k=9.1 \times 10^{9} \mathrm{M}^{-1} \mathrm{~s}^{-1} \\
\bullet \mathrm{H}+\mathrm{N}_{2} \mathrm{O} \rightarrow \bullet \cdot \mathrm{OH}+\mathrm{N}_{2} \quad k=2.1 \times 10^{6} \mathrm{M}^{-1} \mathrm{~s}^{-1}
\end{gathered}
$$




\section{Gamma radiolysis}

Aqueous solutions of BPA were subjected to increasing doses of ${ }^{60} \mathrm{Co}$ gamma radiation and subsequently analyzed for parent compound loss and stable degradation products using HPLC. The initial concentration of BPA varied from 0.01 to $1.4 \mathrm{mM}$ in the deionized water solutions, and was typically $0.5-0.8 \mathrm{mM}$ in treated wastewaters. To determine the removal constant [27], defined as the initial slope of the change in concentration of the compound versus the dose (kGy), only early degradation changes (15-20\% loss) were considered. When solutions of $0.5 \mathrm{mM}$ BPA or greater were utilized, a removal constant of $0.45 \pm 0.04 \mu \mathrm{mol} / \mathrm{kGy}$ was determined. At these relatively high concentrations of BPA, the dissolved substances in the treated wastewaters are simply not competitive with BPA. Therefore, in the early stages of the oxidative degradation in both deionized water solution and treated wastewaters, the removal constant is reflective of the hydroxyl radical's reaction with only bisphenol A. Figure 1 shows the changes in BPA concentrations plotted against the applied doses in $\mathrm{kGy}$ for deionized water and for the treated wastewater described as before membrane filtration (BMF) (Table 1).

When the BPA degradation proceeds beyond $20 \%$, the dissolved substances in the treated wastewaters begin to affect the rate of hydroxyl radical-induced removal of BPA. As the quality of the water decreases, the rate of BPA degradation also diminishes. Figure 2 is a plot of the $\ln \mathrm{Ct} / \mathrm{Co}(\mathrm{Ct}=$ concentration at time $\mathrm{t}$; $\mathrm{Co}=$ original concentration) against applied dose using the treated wastewaters from Orange County's pilot plant: Q1, ROA, and ROB, in addition to deionized and local tap water. Selected water quality parameters for the three post-secondary treated wastewaters are shown in Table 2 . These results shown in the figure demonstrate that

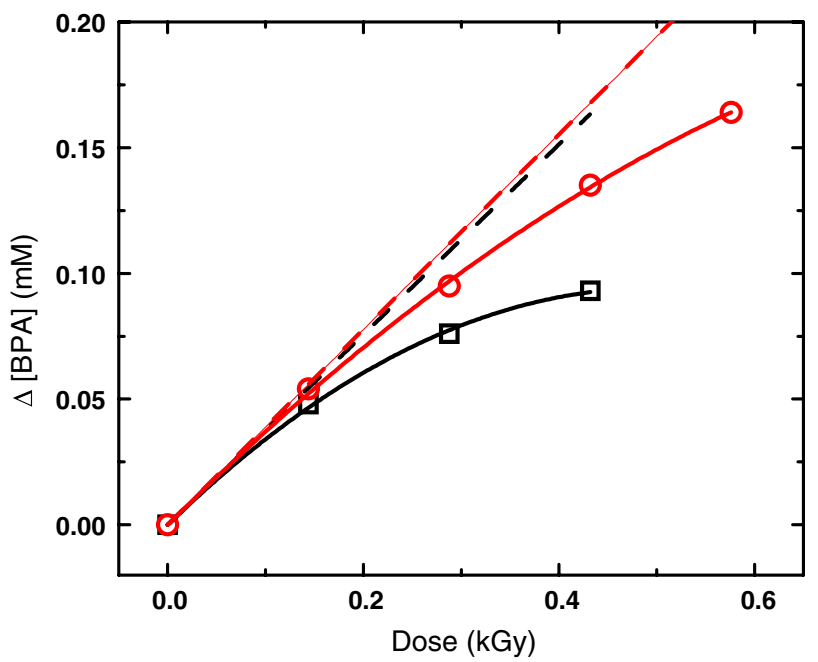

Fig. 1 Dose dependence of concentration change determined for bisphenol A, $0.80 \mathrm{mM}$ in Milli-Q deionized water $(\square)$ and in treated wastewater, $0.80 \mathrm{mM}$ in BMF water (O). Solid lines are fitted quadratic equations, with intercepts fixed at zero. The initial slopes (dashed lines) of these two curves are the corresponding removal constants 


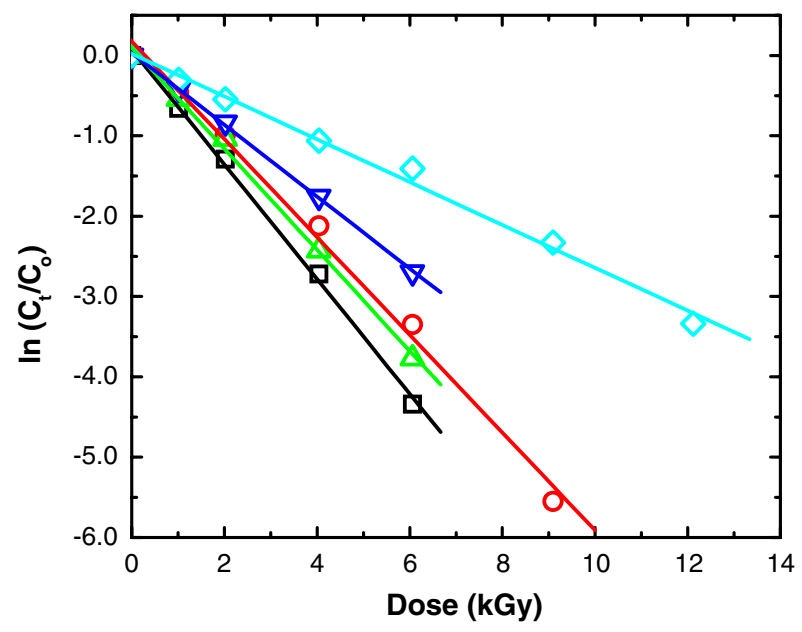

Fig. 2 Plot of $\ln \left(\mathrm{C}_{\mathrm{t}} / \mathrm{C}_{\mathrm{o}}\right)$ against applied radiation dose $(\mathrm{kGy})$ for bisphenol A removal by hydroxyl radicals in waters of different quality. $(\square=$ deionized; $O=$ tap water; $\Delta=\mathrm{ROB} ; \boldsymbol{\nabla}=\mathrm{ROA}$; $\diamond=\mathrm{Q} 1)$

the $\mathrm{OH}$ scavengers present in wastewaters and tap water affect the rate of breakdown of BPA. The lowest quality wastewater (Q1), which contains the greatest amount of hydroxyl radical scavengers, undergoes the slowest BPA breakdown.

In order to further investigate differences in the hydroxyl radical-mediated breakdown of BPA associated with water quality, HPLC analyses of the stable degradation products were performed. The chromatograms of the irradiated BPA deionized water solutions were markedly different from the chromatograms of the irradiated BPA dissolved in BMF treated wastewater, as shown in Fig. 3. The first chromatogram shows a prominent intermediate formed in the hydroxyl radicalmediated oxidation pathways when deionized water was used as the solvent with a radiation dose of $1.3 \mathrm{kGy}$. This intermediate accumulates in the solution and is readily detected throughout most of the irradiation. For the irradiation experiments of BPA in BMF wastewater, this particular stable product is missing, implying that the hydroxyl radical-mediated reaction pathway generates different intermediates which are more competitive with BPA. The HPLC chromatogram for BPA in BMF is displayed as Fig. $3 b$.

The prominent intermediate formed in the gamma irradiation of BPA in deionized water was identified using LC/MS as the hydroxylated BPA, as expected. This product is consistent with common hydroxyl radical reactions with aromatic compounds, where hydroxyl radical addition to the ring occurs [20, 21, 28, 29]. In deionized water, this is clearly a major pathway. Since the hydroxylated BPA accumulates over a period of time before it decreases in concentration, it displays a considerable degree of irradiation stability in the aqueous solution. Other degradation products identified by GC/MS techniques in the hydroxyl radical oxidation include 4-isopropenyl phenol and hydroxylated ring-opened products. These findings correspond to the product analyses reported by Gozmen et al. [30] in 

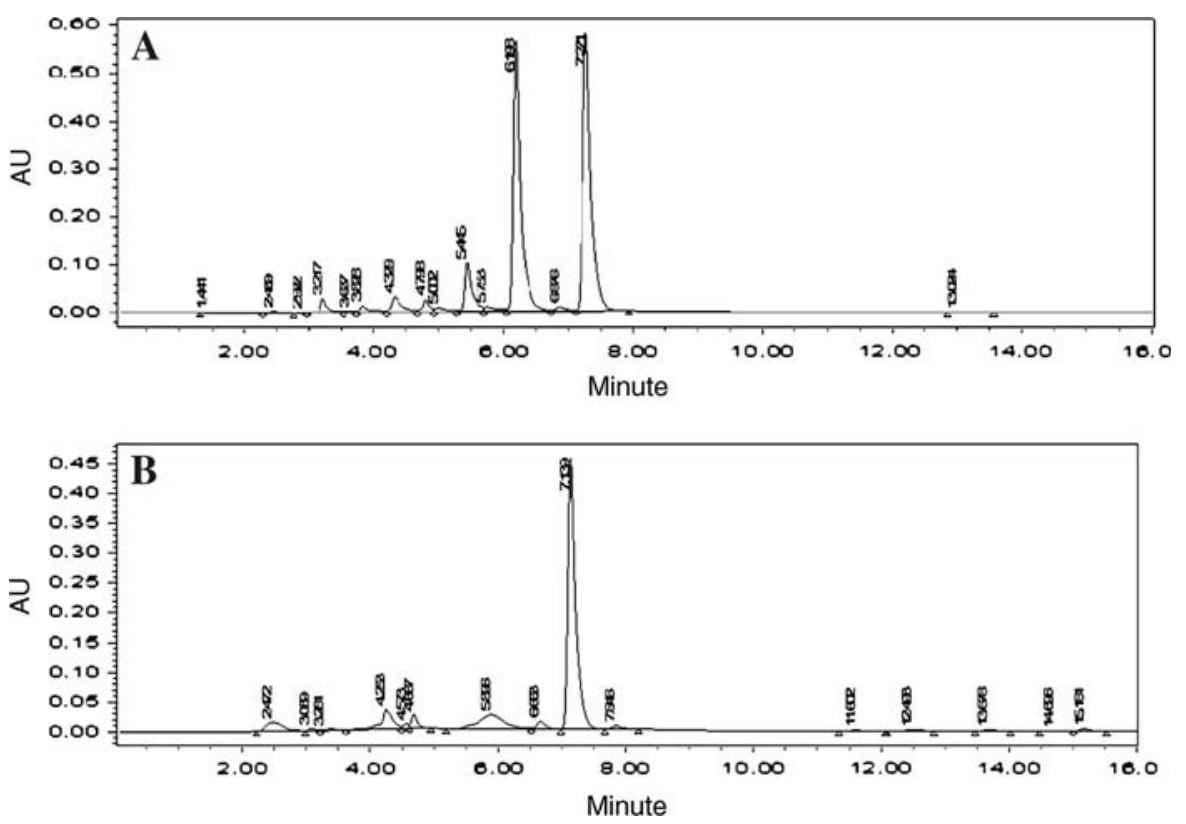

Fig. 3 HPLC chromatograms from irradiated BPA solutions. a Irradiated BPA dissolved in deionized water, dose $=1.3 \mathrm{kGy}$. b Irradiated BPA dissolved in BMF treated wastewater, dose $=1.8 \mathrm{kGy}$. The peak just beyond $7 \mathrm{~min}$ in both chromatograms is BPA. The peak on the top chromatogram (a) at $6.2 \mathrm{~min}$ is the prominent intermediate

the Fenton reagent oxidation of BPA and to the products reported from the peroxidase-mediated oxidation of BPA by Weber and Huang [13]

Solutions of the BPA in BMF wastewater irradiated with different doses were analyzed, but none showed any presence of the hydroxylated bisphenol A. This different reaction pathway is attributed to the presence of other dissolved species in the treated wastewater, which interfere with the BPA hydroxylation reaction. While the hydroxylated BPA accumulates and competes for the hydroxyl radical in the deionized water solution, no such competition takes place in the lower quality treated wastewater solutions.

Pulse radiolysis measurements

To help resolve the different reaction pathways, experiments were conducted using the technique of pulse radiolysis to determine the absolute kinetics of the hydroxyl radical reactions, both in deionized water and in the treated BMF wastewater. Pulse radiolysis experiments using a $0.5 \mathrm{mM}$ bisphenol A solution saturated with nitrous oxide gas in deionized water produced a broad, strongly absorbing transient species centered around $305 \mathrm{~nm}$, as shown in Fig. 4. The maximum absorption for the transient generated from the reaction between the hydroxyl radical and bisphenol $\mathrm{A}$ was found to occur at $\sim 6 \mu \mathrm{s}$. From analyses at $305 \mathrm{~nm}$, pseudo-first-order rate constants were obtained by fitting exponential curves to the pseudo first order 

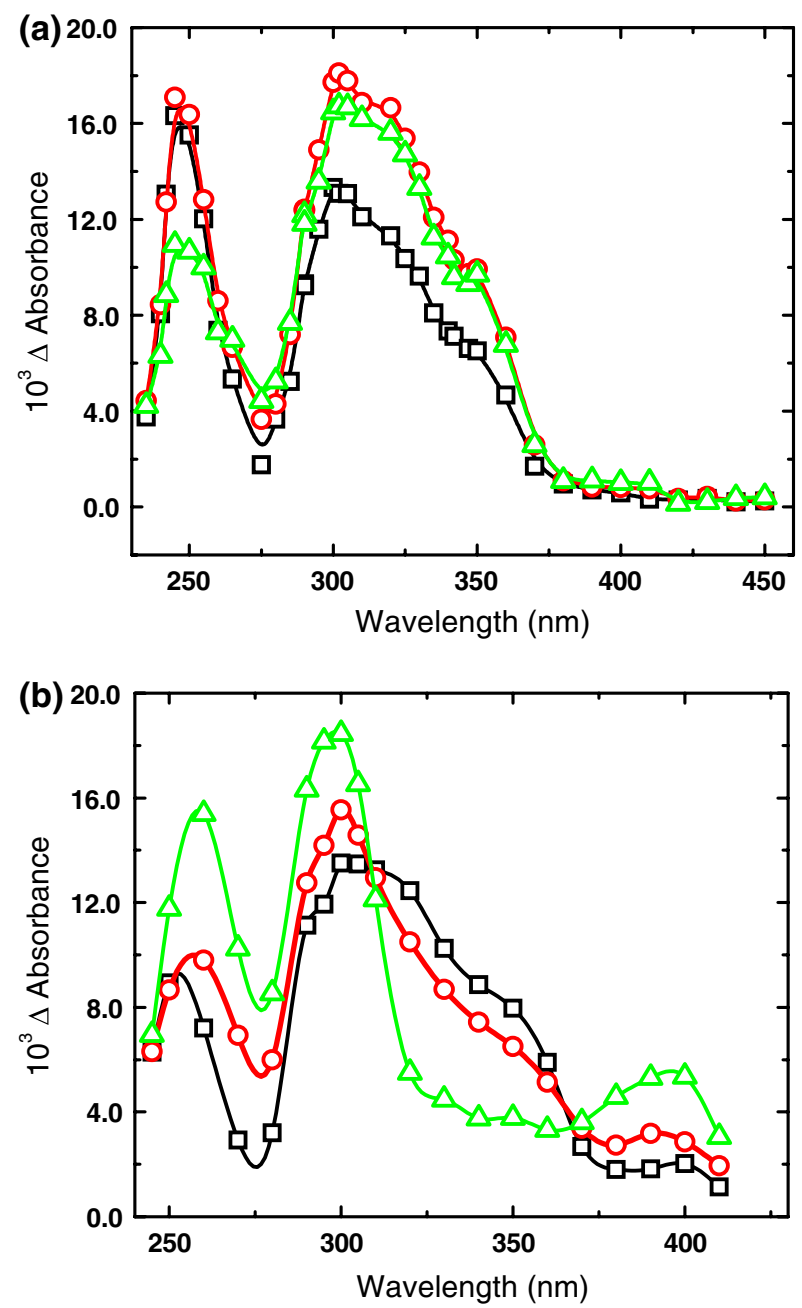

Fig. 4 a Difference transient absorption spectrum formed from the reaction between bisphenol A and the hydroxyl radical in deionized water, recorded at $0.6 \mu$ s ( $\square), 6 \mu$ s (O) and $16 \mu \mathrm{s}(\triangle)$. b Difference transient absorption spectrum formed from the reaction between bisphenol A and the hydroxyl radical in BMF treated wastewater, recorded at $1.5 \mu$ s (black square), $6 \mu$ s (red circle) and $16 \mu \mathrm{s}$ (green triangle)

growth of the transient. These pseudo-first-order rate constant values were plotted for five different concentrations of BPA, and a second order rate constant, $(6.9 \pm 0.2) \times 10^{9} \mathrm{M}^{-1} \mathrm{~s}^{-1}$, was determined from the slope of the line.

The kinetics for an $\mathrm{N}_{2} \mathrm{O}$-saturated solution of $0.8 \mathrm{mM}$ bisphenol A prepared in the BMF treated wastewater showed the same transient absorption at short times (see Fig. 5), but gave a very different transient absorption at longer times. Figure $4 \mathrm{~b}$ displays the transient intensities at $1.5,6$, and $16 \mu$ s after the electron pulse for the hydroxyl radical reaction of BPA in BMF. At $1.5 \mu \mathrm{s}$, the difference transient absorption profile looks similar to the one generated in high purity water. Consistent with the temporal behavior of the spectrum of BPA in DI water at $6 \mu$ s, the 


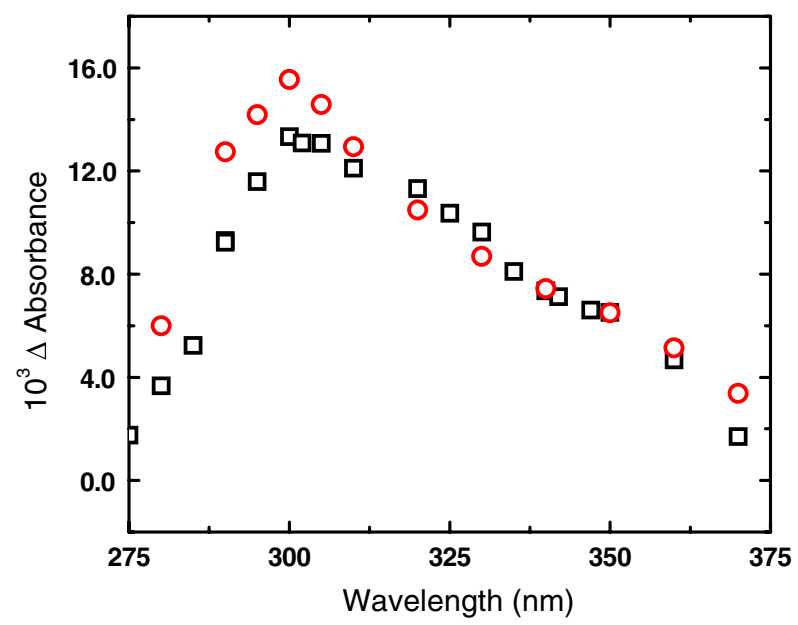

Fig. 5 Difference transient absorption spectra from BPA $+\mathrm{OH}$ at $0.6 \mu$ s in DI water ( $\square$ ) overlayed with the spectrum of $\mathrm{BPA}+\mathrm{OH}$ in $\mathrm{BMF}$ water at $1.5 \mu \mathrm{s}(\mathrm{O})$

absorption at $305 \mathrm{~nm}$ continues to increase for the irradiated wastewater solution. However, over the 6-16 $\mu$ s period, the transient spectrum for $\mathrm{OH}+\mathrm{BPA}$ in BMF water changes noticeably in comparison to the BPA in deionized water. Instead of the slow decrease in absorption seen in Fig. 4a for the BPA in deionized water, the absorption at $305 \mathrm{~nm}$ continues to increase (Fig. 4b) and the peak width narrows. An additional transient growth is noted in the BPA/BMF in the 395-nm region during this period. Transient profiles at $305 \mathrm{~nm}$ were also different over a longer, 16- $\mu \mathrm{s}$, period, as shown in Fig. 6. The transient species detected in the BPA/BMF solution continues to grow on this timescale (Fig. 6a), while the transient at $305 \mathrm{~nm}$ in the BPA/DI solution (Fig. 6b) shows a slight decay.

An even greater discrepancy in the transient absorptions is noted at $347 \mathrm{~nm}$ at longer times. The decay profile at $347 \mathrm{~nm}$ for the bisphenol A transient in DI water is shown in Fig. 7a, and is distinctly different from the growth profile at $347 \mathrm{~nm}$ (Fig. 7b) for the BPA in BMF water. The decay of the pure water solution transient follows the same basic decay pattern seen at the high absorption wavelengths and is typical of transients formed from hydroxyl radical additions to aromatic rings.

The pulse radiolysis data and steady state gamma radiolysis data suggest that the initial oxidative hydroxyl radical reactions are the same for both qualities of water, but that the subsequent chemical pathways involved are distinctly different. The typical clean water pathway for hydroxyl radical reaction with phenolic compounds involves addition of the radical to the aromatic ring to form a transient cyclohexadienyl species [31-33]. This transient has a lifetime on the microsecond timescale and is completely transformed to the stable hydroxylated product by $0.5 \mathrm{~ms}$ (Fig. 7a). This stable hydroxylated intermediate forms and shows some degree of resistance to subsequent hydroxyl radical reactions, since it accumulates as a stable, detectable, intermediate during the oxidation of the bisphenol A. Addition of the hydroxyl radical to bisphenol A and the resultant compound which forms in deionized water are shown in Scheme 1. 

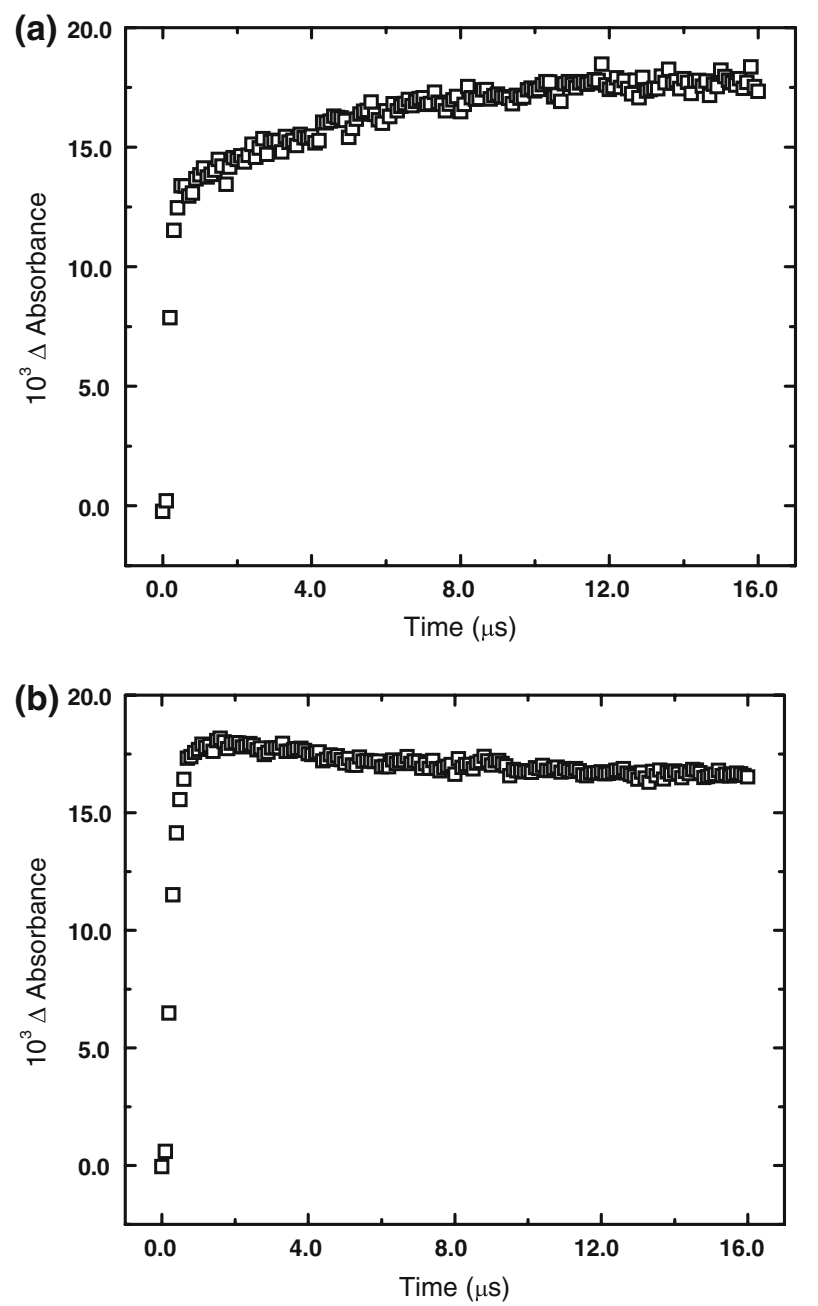

Fig. 6 a Growth profile for BPA $(0.8 \mathrm{mM})+\mathrm{OH}$ in BMF treated wastewater at $305 \mathrm{~nm}$ over $20 \mu \mathrm{s}$. b Growth and decay profile for BPA $(0.5 \mathrm{mM})+\mathrm{OH}$ in deionized water at $305 \mathrm{~nm}$ over same period

In the lower quality wastewater, BMF, subsequent conversion of the transient cyclohexadienyl radical to the stable hydroxylated product is inhibited by a dissolved substance or substances in the treated wastewater. Presently, investigations into the specific substances and the mechanisms involved in the lower quality water solutions which prevent the expected hydroxylated product are in progress.

\section{Conclusions}

The chemical pathways involved in the hydroxyl radical reactions with bisphenol A are significantly affected by the presence of the dissolved substances in typical 

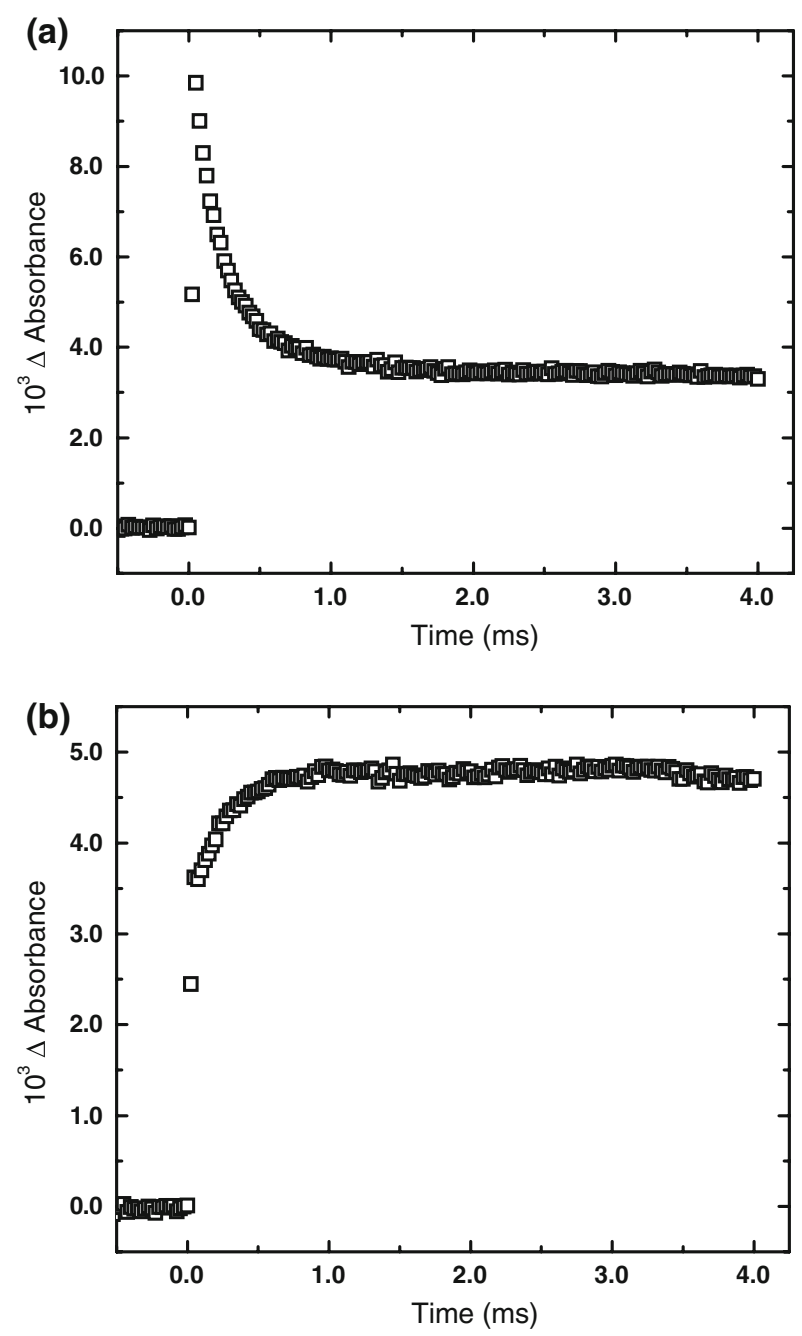

Fig. 7 a Decay profile for BPA reaction with $\mathrm{OH}$ in DI water at $347 \mathrm{~nm}$ over $5 \mathrm{~ms}$. b Growth profile for $\mathrm{BPA}$ reaction with $\mathrm{OH}$ in $\mathrm{BMF}$ wastewater at $347 \mathrm{~nm}$ over $5 \mathrm{~ms}$

tertiary treated wastewaters. The expected hydroxyl radical addition product was not formed when the reaction was conducted in the tertiary treated wastewaters which contain considerable amounts of dissolved substances. The removal constant for the reaction of BPA with the hydroxyl radical was determined at short radiation times, with an overall reaction efficiency for the reaction of BPA $+\mathrm{OH}$ of $76 \%$. The longer irradiation ( $>20 \%$ loss) experiments clearly showed the effects of dissolved substances, where the lowest quality water inhibited the oxidative breakdown of BPA to the greatest extent. Pulse radiolysis results point to a mechanism where the hydroxyl radical attacks the BPA in both pure water and in treated wastewaters to form the cyclohexadienyl radical; however, subsequent 
<smiles>CC(C)(c1ccc(O)cc1)c1ccc(O)cc1</smiles>

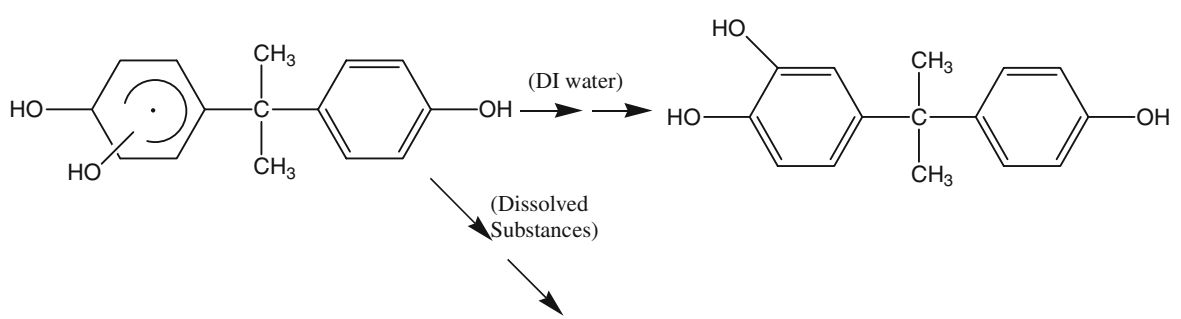

no hydroxylated product

Scheme 1 Resultant compound formed in deniionized water by addition of the hydroxyl radical to bisphenol A

formation of the hydroxylated product only takes place in pure or very highly treated wastewaters.

Acknowledgments Work was performed at the Radiation Laboratory, University of Notre Dame, which is supported by the Office of Basic Energy Sciences, U.S. Department of Energy. Financial support is also acknowledged from the WateReuse Foundation Grant WRF 04-017. We thank Ken Ishida at the Orange County (CA) Sanitation District and Carie Wilson at the City of Scottsdale, Arizona, Water Treatment Plant for wastewater samples and information. This is publication number 18 from the UCI Urban Water Research Center.

Open Access This article is distributed under the terms of the Creative Commons Attribution Noncommercial License which permits any noncommercial use, distribution, and reproduction in any medium, provided the original author(s) and source are credited.

\section{References}

1. R.A. Rudel, S.J. Melly, P.W. Geno, G. Sun, J.G. Brody, Identification of alkylphenols and other estrogenic phenolic compounds in wastewater, septage, and groundwater on Cape Cod, Massachusetts. Environ. Sci. Technol. 32, 861-869 (1998)

2. H.M. Kuch, K. Ballschmiter, Determination of endocrine-disrupting phenolic compounds and estrogens in surface and drinking water by HRGC-(NCI)-MS in the picogram per liter range. Environ. Sci. Technol. 35, 3201-3206 (2001)

3. M. Furhacker, S. Scharf, H. Weber, Bisphenol A: emissions from point sources. Chemosphere 41, 751-756 (2000)

4. H. Fromme, T. Kuchler, T. Otto, K. Pilz, J. Muller, A. Wenzel, Occurrence of phthalates and bisphenol A and F in the environment. Water Res. 36, 1429-1438 (2002)

5. T. Suzuki, Y. Nakagawa, I. Takano, K. Yaguchi, Y. Kazuo, Environmental fate of bisphenol A and its biological metabolites in river water and their xeno-estrogenic activity. Environ. Sci. Technol. 38, 2389-2396 (2004)

6. R. Bannister, N. Beresford, D. May, E.J. Routledge, S. Jobling, M. Rand-Weaver, Novel estrogen receptor-related transcripts in Marisa cornuarietis; a freshwater snail with reported sensitivity to estrogenic chemicals. Environ. Sci. Technol. 41, 2643-2650 (2007)

7. S. Jobling, M. Nolan, C. Tyler, G. Brighty, J.P. Sumpter, Widespread secual disruption in wild fish. Environ. Sci. Technol. 32, 2498-2506 (1998) 
8. L.N. Vandenberg, M.V. Maffini, P.R. Wadia, C. Sonnenschein, B.S. Rubin, A.M. Soto, Exposure to environmentally relevant doses of the xenoestrogen bisphenol-A alters development of the fetal mouse mammary gland. Endocrinology 148, 116-127 (2007)

9. K. Nakamura, K. Itoh, T. Sugimoto, S. Fushiki, Prenatal exposure to bisphenol A affects adult murine neocortical structure. Neurosci. Lett. 420, 100-105 (2007)

10. Y. Takai, O. Tsutsumi, Y. Ikezuki, Y. Kamei, Y. Osuga, T. Yano, Y. Taketan, Preimplantation exposure to bisphenol A advances postnatal development. Reprod. Toxicol. 15, 71-74 (2001)

11. I. Bautista-Toledo, M.A. Ferro-Garcia, J. Rivera-Utrilla, C. Moreno-Castilla, F.J. Vegas Fernandez, Bisphenol A removal from water by activated carbon. Effects of carbon characteristics and solution chemistry. Environ. Sci. Technol. 39, 6246-6250 (2005)

12. G. Belgin, M.A. Oturan, N. Oturan, O. Erbatur, Indirect electrochemical treatment of bisphenol A in water via electrochemically generated Fenton's reagent. Environ. Sci. Technol. 37, 3716-3723 (2003)

13. Q. Huang, J. Weber, Transformation and removal of bisphenol A from aqueous phase via peroxidasemediated oxidative coupling reactions: efficacy, products, and pathways. Environ. Sci. Technol. 39, 6029-6036 (2005)

14. K. Nomiyama, T. Tanizaki, T. Koga, K. Arizono, R. Shinohara, Oxidative degradation of BPA using $\mathrm{TiO}_{2}$ in water, and transition of estrogenic activity in the degradation pathways. Arch. Environ. Contam. Toxicol. 52, 8-15 (2007)

15. R. Thiruvenkatachari, T.O. Kwon, S. Moon, Application of slurry type photocatalytic oxidationsubmerged hollow fiber microfiltration hybrid system for the degradation of bisphenol A (BPA). Sep. Sci. Technol. 40, 2871-2888 (2005)

16. Y. Ohko, I. Ando, C. Niwa, T. Tatsuma, T. Yamamura, Y. Kubota, A. Fujishima, Degradation of bisphenol A in water by $\mathrm{TiO}_{2}$ photocatalyst. Environ. Sci. Technol. 35, 2365-2368 (2001)

17. R.A. Torres, C. Petrier, E. Combat, F. Moulet, C. Pulgarin, Bisphenol A mineralization by integrated ultrasound-UV-iron (II) treatment. Environ. Sci. Technol. 41, 297-302 (2007)

18. M. Deborde, S. Rabouan, J.-P. Duguet, B. Legube, Kinetics of aqueous ozone-induced oxidation of some endocrine disruptors. Environ. Sci. Technol. 39, 6086-6092 (2005)

19. T. Saito, K. Kato, Y. Yokogawa, M. Nishida, N. Yamashita, Detoxification of bisphenol A and nonylphenol by purified extracellular laccase from a fungus isolated from soil. J. Biosci. Bioeng. 98, 64-66 (2004)

20. G. Buxton, C. Greenstock, W.P. Helman, A.B. Ross, Critical review of rate constants for reactions of hydrated electrons, hydrogen atoms and hydroxyl radicals $\left(\mathrm{OH} / \mathrm{O}^{-}\right)$in aqueous solution. J. Phys. Chem. Ref. Data 17, 513-886 (1988)

21. J. Peller, P.V. Kamat, Radiolytic transformations of chlorinated phenols and chlorinated phenoxyacetic acids. J. Phys. Chem. A 109, 9528-9535 (2005)

22. M.J. Lundqvist, L.A. Eriksson, Hydroxyl radical reactions with phenol as a model for generation of biologically reactive tyrosyl radicals. J. Phys. Chem. B 104, 848-855 (2000)

23. U. Stafford, K. Gray, P.V. Kamat, Radiolytic and $\mathrm{TiO}_{2}$-assisted photocatalytic degradation of 4chlorophenol. A comparative study. J. Phys. Chem. 98, 6343-6351 (1994)

24. R. Terzian, N. Serpone, Pulse radiolytic studies of the reaction of pentahalophenols with OH radicals: formation of pentahalophenoxyl, dihydroxylpentahalocyclohexadienyl, and semiquinone radicals. Langmuir 7, 3081-3089 (1991)

25. K. Whitham, S. Lyons, R. Miller, D. Nett, P. Treas, A. Zante, R.W. Fessenden, M.D. Thomas, Y. Wang, Linear accelerator for radiation chemistry research at Notre Dame in IEEE Proceedings of the Particle Accelerator Conference and International Conference on High Energy Accelerators, Dallas, Texas, 1995, pp. 131-133

26. G.V. Buxton, C.R. Stuart, Re-evaluation of the thiocyanate dosimeter for pulse radiolysis. J. Chem. Soc. Faraday Trans. 91, 279 (1995)

27. S.P. Mezyk, T. Neubauer, W.J. Cooper, J.R. Peller, Free-radical-induced oxidative and reductive degradation of sulfa drugs in water: absolute kinetics and efficiencies of hydroxyl radical and hydrated electron reactions. J. Phys. Chem. A 111, 9019-9024 (2007)

28. G. Albarran, J. Bentley, R.H. Schuler, Substituent effects in the reaction of OH radicals with aromatics: toluene. J. Phys. Chem.A 107, 7770-7774 (2003)

29. M. Al-Sheikhly, J. Silverman, P. Neta, L. Karam, Mechanisms of ionizing radiation-induced destruction of 2,6-dichlorophenyl in aqueous solutions. Environ. Sci. Technol. 31, 2473-2477 (1997)

30. B. Gozmen, M.A. Oturan, O. Oturan, O. Erbatur, Indirect electrochemical treatment of bisphenol A in water via electrochemically generated Fenton's reagent. Environ. Sci. Technol. 37, 3716-3723 (2003) 
31. M. Roder, L. Wojnarovits, G. Foldiak, S.S. Emmi, G. Beggiato, M. D’Angelantonio, Addition and elimination kinetics in $\mathrm{OH}$ radical induced oxidation of phenol and cresols in acidic and alkaline solutions. Radiat. Phys. Chem. 54, 475-479 (1999)

32. N.V. Raghavan, S. Steenken, Electrophilic reaction of the $\mathrm{OH}$ radical with phenol. Determination of the distribution of isomeric dihydroxycyclohexadienyl radicals. J. Am. Chem. Soc. 102, 3495-3499 (1980)

33. G. Buxton, J.R. Langan, J.R. Smith, Aromatic hydroxylation. 8. A radiation chemistry study of the oxidation of hydroxycyclohexadienyl radicals. J Phys. Chem. 90, 6309-6313 (1986) 